\title{
Memorias de la Escuela Nacional de Enfermería y Obstetricia ENEO-UNAM
}

\section{Memories of National School of Nurse and Obstetrics ENEO-UNAM}

Maestra Iñiga Pérez Cabrera

Decana de la Escuela Nacional de Enfermería y Obstetricia de la ENEO•UNAM

Los grandes descubrimientos de finales del siglo XIX en el campo de la física y la química transformaron la visión y la interpretación de lo que ocurre en el interior del cuerpo humano. Al tener acceso a los nuevos conocimientos científicos, las profesiones estuvieron condicionadas a demostrar su actividad por medio de la tecnología en el servicio prestado a la sociedad, de esta forma, nace el monopolio del saber acerca de la enfermedad y la salud en el caso de la profesión médica (Colliere, 1995).

Al mismo tiempo, en el Hospital de San Andrés de la Ciudad de México, el grupo médico hizo un gran esfuerzo por tecnificar a las personas que se interesaban en la Enfermería, en donde se adiestró por decreto a un grupo de mujeres, que posteriormente se harían cargo del servicio de pacientes en pabellones del Hospital General de México, que hoy conocemos como jefes de sala (Pérez-Loredo, 2008).

En virtud de que en México aún se carecía de enfermeras idóneas para la enseñanza, se realizaron los trámites con personalidades médi- cas y diplomáticas de Estados Unidos, con quienes el Doctor Liceaga conservaba amistad, con el fin de contratar a diversas enfermeras americanas por cortos periodos, para hacerse cargo del adiestramiento de las enfermeras mexicanas. Estas lecciones fueron una novedad en la época, establecieron un ordenamiento técnico-médico para que las enfermeras se capacitaran en diversos aspectos hospitalarios, y para que se iniciara la conformación de una enseñanza formal que diera nacimiento a la imagen de la enfermera de principios del siglo XX.

El 9 de febrero de 1907, dos años después de haberse inaugurado el Hospital General de México, por solicitud expresa al presidente Porfirio Díaz, se inició la institucionalización de la primera escuela de Enfermería en México, la del Hospital General, que marcó una tendencia de formalización de los programas educativos y la oficialidad de sus certificados en control de interés público. Esa escuela fue el antecedente de lo que hoy es la Escuela Nacional de Enfermería y
Obstetricia; a partir de esa fecha, las enfermeras llegaron a los servicios con calificaciones en aspectos técnicos y humanos, a la altura de lo que se requería.

Una vez inaugurada la Universidad Nacional de México en 1910, al año siguiente aprobó la integración de la enseñanza de la Enfermería a la Escuela de Medicina, una oportunidad para salir del espacio unidimensional del Hospital-Escuela. En 1912, los médicos diseñaron el primer plan de estudios para cursar en dos años la Carrera de Enfermera y dos años para la Carrera de Partera.

En 1922, en la época posrevolucionaria, ya en plena reconstrucción del país y fundada la Secretaria de Educación Pública,..."los planes de estudio, tanto la Carrera de Enfermera como la de Partera fueron solicitados por las escuelas incorporadas del interior del país, a las autoridades de la Universidad Nacional de México, unificándose así la enseñanza de la enfermería" (Bermúdez: 2008). Visto desde el campo de trabajo, 
el desarrollo de la profesión se dio en tres vertientes: partera, enfermera sanitaria y enfermera hospitalaria o asistencial.

De 1911 a 1926 la formación profesional de enfermeras requirió para su ingreso los estudios de primaria superior. En 1927, dos años después de haberse implantado la enseñanza secundaria en el sistema educativo de México, se estableció cursar un ciclo preparatorio de un año, impartido en la Escuela Nacional Preparatoria, en el que se estudiaba: biología, matemáticas, química y física, asignaturas que orientaban la mejor comprensión de los conocimientos de la carrera de Enfermera.

En 1929, año de la Autonomía Universitaria de la UNAM, otorgada por el Presidente de la República Emilio Portes Gil, se reestructuró el plan de estudios de la carrera de Enfermera y el de Partera. A partir de 1938, se les exigió a las aspirantes la presentación de su comprobante de estudios secundarios, para obtener la inscripción a la carrera de enfermera y haber cursado la misma, para la carrera de Partera.

De 1936 a 1940, las políticas de salud, dieron mayor auge a la construcción de hospitales, y en la década de los cuarenta se crearon los de especialidad, esto trajo consigo cambios en la atención a los pacientes y mayor demanda de enfermeras. Con esto mejoraron las condiciones laborales y económicas (Ávila, 1986). Ante este escenario, en 1944 se modificó el plan de estudios, incrementándose el número de asignaturas de cuatro a diecisiete, para la Carrera de Enfermera, materias que se iban perfilando a las nuevas necesidades de las especialidades, y a la carrera de

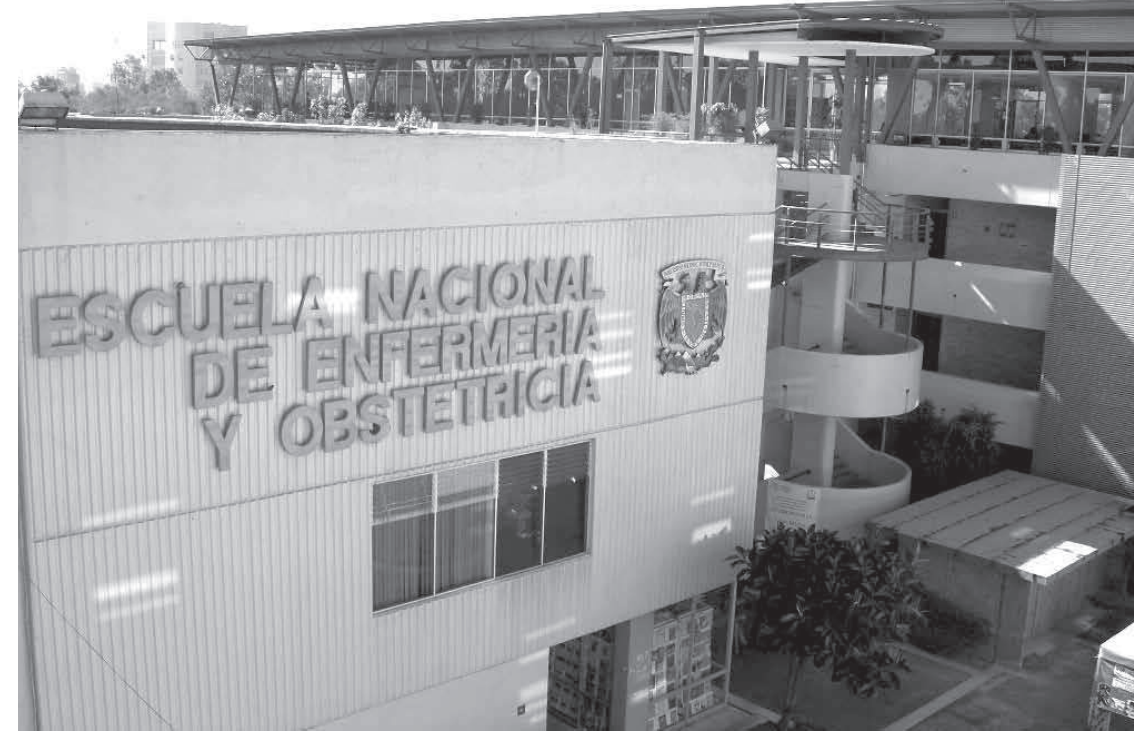

Partera se le agregó la asignatura de Puericultura (Pérez \& Castañeda, 2006).

La Ley Orgánica de 1945 define a la UNAM, como un organismo descentralizado del Estado, que le asigna la finalidad de realizar las funciones sustantivas de docencia, investigación y extensión de la cultura. Instituye el fundamento de las carreras universitarias al constituir en cada entidad académica, una estructura legal que se sustenta en la autonomía, a través de los $\mathrm{H}$. Consejos Técnicos. En este marco legal nació la Escuela Nacional de Enfermería y Obstetricia (ENEO), bajo la dirección de los médicos con orientación biologicista y de Salud Pública. El primer director fue el Dr. Everardo Landa, quien pugnó porque las enfermeras participaran directamente en la formación de las nuevas generaciones. En 1948, la Dra. Emilia Leija Paz de Ortiz consiguió que las oficinas para la dirección y la administración de la Escuela se instalaran en el Pasaje Catedral en el Centro Histórico de la Ciudad de México.

En 1952, se creó el primer curso de Enfermeras Instructoras, auspiciado por la Universidad Nacional Autónoma de México y la Organización Mundial de la Salud. En 1953, se aprobó en sesión del Consejo Universitario, la solicitud presentada por el H. Consejo Técnico y por la sociedad de alumnos de la misma escuela de: "Considerar a las egresadas de la Escuela Nacional de Enfermería y Obstetricia como "universitarias" ya que el requisito para ingresar a la escuela sería la Preparatoria (oficio No. 0651)". En este mismo año, la Organización Panamericana de la Salud (OPS) estableció un convenio con México, con el fin de realizar el proyecto de un nuevo plan de estudios de la Escuela Nacional de Enfermería y Obstetricia, y satisfacer las peticiones hechas por las enfermeras mexicanas. Este proyecto se consolidó en el "Seminario de Educación de Enfermería en América Latina" realizado en Zimapán, Hidalgo. El objetivo de éste fue "Estudiar la situación real de los servicios de enfermería del país, para que la preparación del personal se basara en las necesidades existen- 
tes, y conseguir el mejoramiento de los servicios de enfermería en toda la América Latina" (Pérez \& Castañeda, 2006: 27).

En 1961, durante la gestión del Dr. Alfredo López de Nava, consiguió como sede para la ENEO, el edificio de la antigua Facultad de Medicina y realizó el primer intento de exigir Bachillerato como requisito de ingreso a la ENEO. El Dr. Lázaro Pavía Crespo, al ocupar la dirección de la escuela, por vez primera nombró a una enfermera, Juventina Hernández Márquez, como Secretaria General, se otorgaron nombramientos a las instructoras de acuerdo con su preparación, las equivalencias para la revalidación de estudios profesionales fueron establecidas, y los exámenes profesionales de escuelas incorporadas se realizaron en la ENEO. También, adquirió equipo para el laboratorio de prácticas de enfermería.

El 15 de julio de 1963, se otorgó el nombramiento de subdirectora a la enfermera Delia Otero Miranda y de secretario al Doctor Federico Bravo Peña. En 1964 se inauguró el Curso de Educación en Enfermería para Enfermeras Instructoras en la ENEO. Después le siguieron otros más hasta la década de los ochenta. La ENEO continuó su evolución y progreso; el director Dr. Rafael Tovar Villagordoa contó con colaboradoras magníficas, entre ellas, las profesoras: Ma. de Jesús Morales Herrera, Delia Otero Miranda y Juventina Hernández Márquez.

En 1966, con el Dr. Alejandro Guevara Rojas, de acuerdo a los cambios de la Reforma Universitaria, se adoptó el sistema de créditos, se estructuró el plan de estudios por semestres y, finalmente se hicieron los trámites para la aprobación de la licenciatura en Enfermería y Obstetricia, el 15 de febrero de 1968.

En 1975, por primera vez en la historia de la ENEO, una enfermera dirigió los destinos de la escuela, la Licenciada Marina Guzmán Vanmeeter (1975-1982), durante su gestión se evaluó el currículo de las Carreras de Enfermera y de la Licenciatura en Enfermería y Obstetricia, con la asesoría de la OMS/ OPS y la participación de profesoras de la ENEO, de las escuelas incorporadas, de los alumnos y del personal de salud de las instituciones, con el objeto de reestructurar el plan de estudios para la carrera de Enfermera y la de Licenciado en Enfermería y Obstetricia. El Consejo Universitario aprobó el Curso Especial Transitorio para obtener el grado de Licenciado, con el fin de regularizar a las egresadas de nivel técnico y el servicio social para ambas carreras. En 1976 inició el Sistema de Universidad Abierta para la carrera de Enfermera. En 1979, el rector de la UNAM Guillermo Soberón Acevedo inauguró las nuevas instalaciones de la ENEO. También, con motivo del cincuentenario de la Autonomía Universitaria, se elaboró la cronología de la Escuela Nacional de Enfermería y Obstetricia. En 1982, los folletos de orientación a la carrera mostraban lo siguiente: "que los grandes problemas de salud por los que el país atraviesa representa una gran responsabilidad para la ENEO, ya que debe preparar profesionales altamente calificados, desde el punto de vista académico como humano, que respondan con conciencia social ante estas necesidades."

En 1983, la Licenciada en Enfermería Esther Hernández Torres
(1982-1986) presentó una propuesta, y su aprobación fue la acción concreta a la recomendación del Rector de la UnAm Dr. Octavio Rivero Serrano, quien demandó incrementar los índices de titulación y la eficiencia terminal, lo que al mismo tiempo favoreció el reconocimiento de los licenciados en el campo laboral. La Coordinación de Investigación orientó sus acciones a la formación de alumnos y docentes en metodología de investigación. Se elaboró el documento "Evaluación de las prácticas clínicas”, ante la problemática que se presentaba en esta actividad. En 1985, se celebró la conmemoración de los 75 años de la apertura de la UNAM y. en 1986 los 75 años de la Enfermería Universitaria, con cuyos trabajos fue realizada la publicación: "Reseña histórica de la creación y evolución de la ENEO, 1911-1986."

En 1987, con la Maestra Graciela Arroyo de Cordero como directora (1987-1994) se llevó a cabo, el primer examen por áreas de conocimiento como opción de titulación, en los dos niveles: técnico y licenciatura y de los dos sistemas, con incremento sustancial de la eficiencia terminal. En 1988 se inició en la ENEO, la impartición de la licenciatura en el Sistema de Universidad Abierta, con la complementación de créditos previa revalidación de los estudios técnicos, para quien hubiera cursado la Carrera de Enfermera y cubriera el requisito de bachillerato. En 1990, del 16 al 18 de octubre, la ENEOUNAM participó como Organismo Patrocinador en el II Coloquio Panamericano de Investigación en Enfermería, en el Palacio de Medicina del Centro Histórico de la Ciudad de México, cuyo tema cen- 
tral fue: "Modelos Alternativos de Enfermería en la Transformación de los Servicios y la Salud de las Poblaciones." En este periodo, la investigación estuvo orientada a la investigación educativa, en apoyo a la evaluación del Plan de Estudios. Se integró el registro sistemático de los informes de investigación; además se editaron los catálogos: "Trabajos de Investigación elaborados por docentes y alumnos de la ENEO (1975 a 1989)" y el de "Investigaciones realizadas por los académicos de la ENEO en el periodo 1987-1994", mismos que quedaron respaldados por el acervo de los informes de la mayoría de los estudios realizados en estos periodos.

El 11 de marzo de 1992, fue aprobado por el H. Consejo Universitario el Plan de Estudios de la Licenciatura en Enfermería y Obstetricia específico de este nivel, previo análisis evaluativo de planes de estudios y perfiles académicos que lo sustentaron. El 7 de abril de 1992, fue inaugurada la biblioteca por el Rector Dr. José Sarukhán Kermez, día en que rindió el primer informe de labores de su segundo periodo en la dirección la Mtra. Arroyo de Cordero. En 1993-1994, con el Programa de regularización de la planta docente, los profesores de asignatura pasaron a profesores de carrera, lo cual contribuyó a desarrollar las expectativas más allá de las esperadas al incrementarse las plazas de profesor de carrera del 6.5 por ciento al 20 por ciento.

En 1997, en la gestión de la Lic. en Enfermería Susana Salas Segura (1995-2002), la ENEO fue nombrada como Centro Colaborador de la Organización Mundial de la Salud para el desarrollo de la Enfermería profesional. En esa mis- ma fecha, iniciaron los estudios de posgrado con el Plan Único de Especialidades en Enfermería (PUEE), y se creó el Comité de investigación. En 2002, la ENEO tuvo a su cargo la organización del VIII Coloquio Panamericano de Investigación en Enfermería, con apoyo del Consejo Nacional de Ciencia y Tecnología (CONACYT), la Oficina Panamericana de la Salud (OPS), así como los Centros Colaboradores de las universidades de Michigan, California, San Francisco, George Mason y el de Alberta Canadá. El tema central fue "La Construcción de Redes de Investigación por la Salud". En la Gaceta Universitaria se informó del evento con el título: "Presentan 227 trabajos en el coloquio de la ENEO".

Acorde con las propuestas de la Organización Mundial de la Salud (OMS), desde la formación académica de los futuros profesionales de Enfermería, estos deben investigar para contribuir a que la población alcance estilos de vida saludables y una atención adecuada, tuvo lugar el XVIII Encuentro Nacional de los
Estudiantes de Enfermería "Avances de la Investigación de Enfermería en el Pregrado", realizado en el Palacio de Medicina, de la Ciudad de México, el 14 y 15 de noviembre de 2002. En éste se presentaron 83 trabajos: 28 en la modalidad oral y 55 en cartel. En este mismo periodo se aprobó la Maestría en Enfermería por el Consejo Académico del Área de las Ciencias Biológicas y de la Salud (CAABYS).

En 2003, asumió la dirección de la ENEO el Lic. Severino Rubio Domínguez (2003-2010), etapa en la cual ha tenido gran impulso el fortalecimiento de la carrera académica y la consolidación de la Investigación Institucional, el desarrollo de la formación de los recursos humanos acorde a las necesidades del país, y a su vez la educación permanente de las enfermeras de los servicios de salud, tanto en su actualización profesional como en el crecimiento académico, que les ha permitido el ascenso en su carrera laboral en forma continua.

Diversas estrategias han permitido la consolidación de la in-

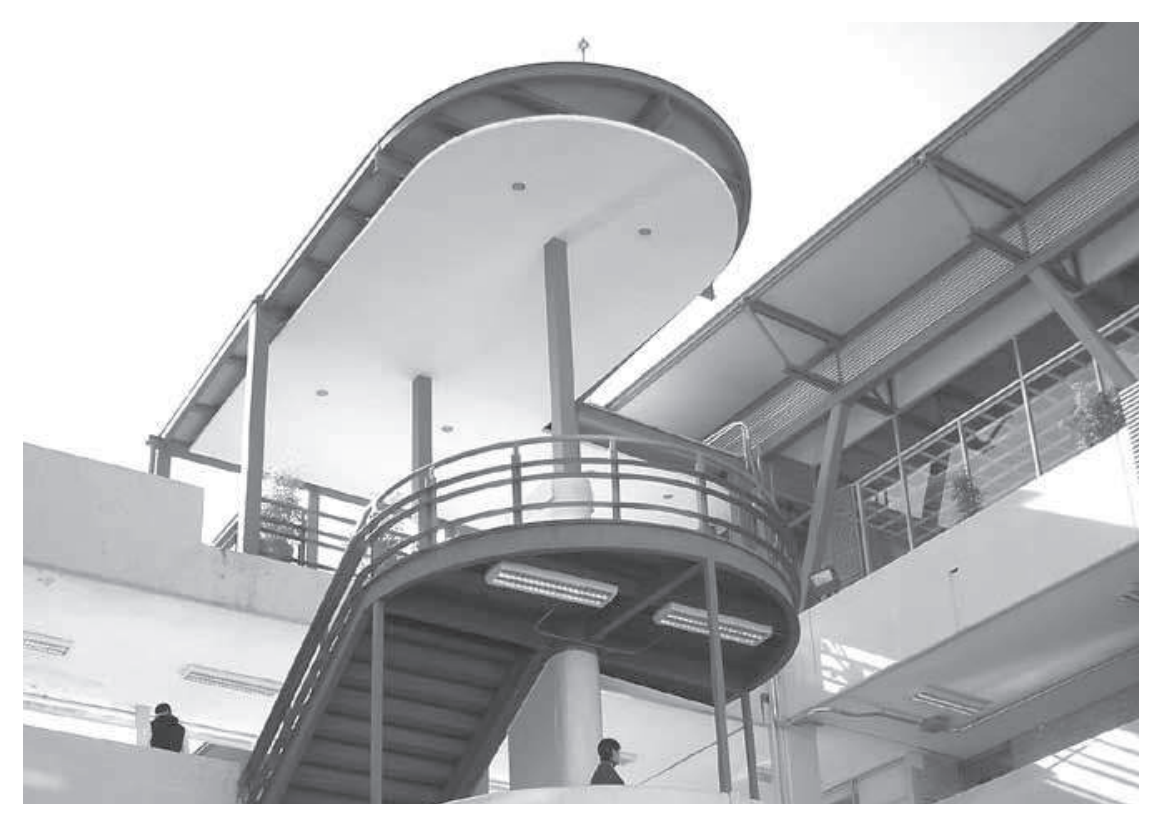


vestigación, unas orientadas al desarrollo de las habilidades investigativas de los docentes, otras a la promoción de proyectos de investigación para fortalecer los vínculos enseñanza-servicio-sociedad, el fortalecimiento del sistema de investigación institucional con la búsqueda de convenios y financiamientos con instituciones nacionales y extranjeras, la actualización de la organización y funcionamiento del Comité de Investigación con base en el Plan de Desarrollo Institucional 2003-2013; y con la participación de los docentes en el Primer Congreso sobre la Investigación en las Facultades y Escuelas de la UNAM. La difusión de productos científicos en la actividad editorial de la ENEO, a través de la edición del Boletín Acontecer Académico, de la Revista Enfermería Universitaria, además de la participación en el Mirador Universitario con barra de programas para transmisión por EDUSAT y Canal 22.

En 2005, los Comités Interinstitucionales de Evaluación de EducaciónSuperior:(CIEES) leotorgaronel nivel 1, del Padrón de calidad educativa a la Licenciatura en Enfermería y Obstetricia y, el Consejo Mexicano de Acreditación y Certificación de la Enfermería, A. C. (COMACE) la Acreditación a la licenciatura en Enfermería y Obstetricia.

En el 2006, se programaron diversas actividades científicas y culturales como parte del programa de conmemoración de los 100 años de formación profesional de las enfermeras. El 9 de febrero de 2007, en la ENEO se celebró con una magna ceremonia, a la que asistió el rector Dr. Juan Ramón de la Fuente, en la que se presentó el CD

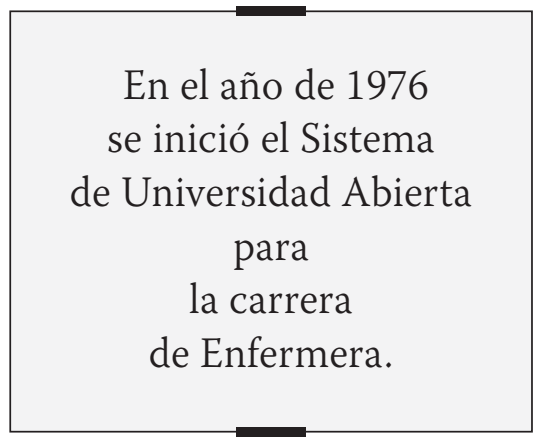

“100 años de la ENEO 1907-2006", ese mismo día, fueron inaugurados los laboratorios de las ciencias básicas después de su remodelación y equipamiento, así como la Unidad de Investigación, misma que conmemora año con año este hecho, con la Jornada de investigación, en la que se presentan los proyectos e informes de investigaciones realizadas por el personal académico de la ENEO.

La remodelación de los laboratorios de enseñanza clínica y el equipamiento de estas áreas, han permitido un fuerte apoyo a la práctica de las tecnologías del cuidado para los estudiantes, de manera que puedan incrementar su habilidad y el mejor desempeño en los campos de experiencia clínica, así como en los procedimientos de Resucitación Cardio-Respiratoria (RCP avanzado), dado los equipos ex profeso con los que cuenta la ENEO.

También, La Biblioteca "Graciela Arroyo de Cordero" ha sido remodelada y equipada con mobiliario nuevo, lo que permitió evaluar los acervos y planear las áreas acorde a la innovación de los recursos informáticos. Nuevas espacios están en proceso de construcción considerando el incremento de la población escolar. y el desarrollo de los programas estratégicos del Plan de Desarrollo Institucional, así como el equipamiento de una nueva área de cómputo para los estudiantes, la apertura de la librería en una área accesible para la exposición y venta de la producción editorial de la ENEO.

Gran logro es la implementación de la Licenciatura en Enfermería en el ciclo escolar 2010-1, además de la Licenciatura en Enfermería y Obstetricia que se encuentra en proceso de actualización de los programas, y de inicio de un proyecto de reestructuración, acorde a las necesidades del país, dados los índices de mortalidad materna.

Con estos avances y logros, la aspiración de la Enfermería Universitaria Mexicana iniciada con la apertura de la Universidad Nacional de México, y próxima a cumplir los 100 años en el 2011, ha experimentado un notable desarrollo similar al que se está dando en Latinoamérica en los últimos años. La investigación en la enfermería aunada a los valores de respeto al ser humano, cobra un lugar preponderante al estar centrada en el proceso de cuidar, de educar y de fundamentar científicamente la toma de decisiones, con el sustento de principios filosóficos, en la bioética y en la historia de enfermería, que fortalece al mismo tiempo la identidad con la profesión. Esta sólida base científica, sin duda promoverá mejores resultados en el ejercicio de una práctica fundamentada en la ciencia y el arte del cuidado, y que el acto de cuidar sea una actividad reflexiva, deliberada y autónoma con reconocimiento legal que reditué en la calidad de este mismo, y proporcione plena seguridad a los demandantes de los cuidados para promover la vida, recuperar la salud o acompañar en la fase terminal. 